\title{
Towards the characterization of the hot Neptune/super-Earth population around nearby bright stars
}

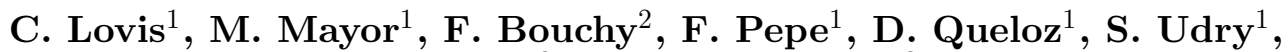 \\ W. Benz ${ }^{3}$ and C. Mordasini ${ }^{3}$ \\ ${ }^{1}$ Observatoire de Genève, Université de Genève, \\ $51 \mathrm{ch}$. des Maillettes, CH-1290 Sauverny, Switzerland \\ email: christophe.lovis@obs.unige.ch \\ ${ }^{2}$ Institut d'Astrophysique de Paris, \\ 98bis Bd Arago, F-75014 Paris, France \\ ${ }^{3}$ Physikalisches Institut, Universität Bern, \\ Sidlerstrasse 5, CH-3012 Bern, Switzerland
}

\begin{abstract}
The HARPS search for low-mass extrasolar planets has been ongoing for more than 4 years, targeting originally about 400 bright FGK dwarfs in the solar neighbourhood. The published low-mass planetary systems coming from this survey are fully confirmed by subsequent observations, which demonstrate the sub-m/s long-term stability reached by HARPS. The complex RV curves of these systems have led us to focus on a smaller sample of stars, accumulating more data points per star. We perform a global search in our data to assess the existence of the large population of ice giants and super-Earths predicted by numerical simulations of planet formation. We indeed detect about 45 candidates having minimum masses below $30 \mathrm{M}_{\oplus}$ and orbital periods below 50 days. These numbers are preliminary since the existence of these objects has to be confirmed by subsequent observations. However, they indicate that about $30 \%$ of solar-type stars may have such close-in, low-mass planets. Some emerging properties of this low-mass population are presented. We finally discuss the prospects for finding transiting objects among these candidates, which may possibly yield the first nearby, transiting super-Earth.
\end{abstract}

\section{Introduction}

HARPS is a high-resolution, fiber-fed echelle spectrograph optimized for high-accuracy radial velocity $(\mathrm{RV})$ measurements. It is mounted on the ESO-3.6m telescope at La Silla Observatory, Chile. Since 2003, the HARPS consortium has been conducting a highprecision search for low-mass extrasolar planets around a sample of about 400 bright, nearby FGK dwarfs. The stars have been selected for having low projected rotational velocities and activity levels, thus minimizing the stellar noise affecting precise RV measurements. The achieved long-term RV precision below $1 \mathrm{~m} / \mathrm{s}$ allows us to detect planets mainly composed of heavy elements such as ices, silicates and iron, as opposed to the more massive gas giants which can be detected at lower precisions. In fact, the difference between precisions of 3 and $1 \mathrm{~m} / \mathrm{s}$ is not merely of technical nature; it is rather a qualitative step forward in our understanding of planetary systems in that it reveals a whole new population of extrasolar planets.

\section{Early results and observational challenges}

Since 2004 several low-mass planetary systems have been published based on HARPS radial velocity measurements. We recall here $\mu$ Ara (HD 160691, Santos et al. 2004, 
Pepe et al. 2007), HD 4308 (Udry et al. 2006) and HD 69830 (Lovis et al. 2006), which were part of the high-precision program described above. We have continued to monitor these stars to refine the orbital parameters and check for the presence of other bodies in the systems. In all cases, the published orbital solutions are fully confirmed by subsequent observations.

For example, for the HD 69830 system, we have now gathered about twice as many data points as the published RV data. The three RV signals are still clearly present in the periodogram and the updated orbital solution differs only marginally from the published one. The dispersion of the residuals around the fit amounts to only $90 \mathrm{~cm} / \mathrm{s}$, demonstrating that HARPS reaches sub-m/s accuracy over more than 4 years.

The recent detections of low-mass planets have shown that these objects are very often found in systems. This gives rise to very complex, low-amplitude radial velocity curves which are not easy to resolve. The optimal observing strategy for a given star is a priori unknown, given that the relevant timescales (i.e. the orbital periods) can extend over three orders of magnitude. These characteristics have led us to change our observing strategy, focusing on a smaller number of targets but accumulating more measurements per target. We typically follow newly observed stars every night during an observing run (7-10 consecutive nights). If high-frequency variations seem to be present, we continue the monitoring at the same cadence in subsequent observing runs. Otherwise, the measurement frequency is decreased and adapted to possible variations at longer timescales.

\section{An emerging population of hot Neptunes and super-Earths}

The presently observed distribution of extrasolar planet masses shows two interesting features (see Fig. 1): a planet "desert" at intermediate masses $\left(\sim 30-100 \mathrm{M}_{\oplus}\right)$ and a hint at an increase in population below $20-30 \mathrm{M}_{\oplus}$. This hint is actually very significant given the detection bias of the RV technique towards lower masses. This bimodal character of the mass distribution, which has to be confirmed by more discoveries of low-mass planets, is in agreement with the predictions of population synthesis simulations based on the core-accretion scenario (see e.g. Mordasini et al. 2008).

In the hope of detecting this new planet population, we performed a systematic search for low-mass bodies in our HARPS high-precision sample. At present not all the stars have been observed sufficiently to perform a complete analysis, so the results are still preliminary. We have found about 45 candidates with minimum masses below $30 \mathrm{M}_{\oplus}$ and orbital periods below 50 days. The plausibility of the orbits has been assessed using periodogram analysis and F-tests. However, these diagnostics are of limited value given the sometimes low number of measurements and the non-gaussianity of the errors. Some human judgement is unavoidable in the selection of the candidates. More data points will tell whether the RV signals are real.

Fig. 2 shows two new low-mass planetary systems coming from this list of candidates, for which the orbital solutions are already well constrained. They are described in details in two recently submitted papers (Mayor et al. 2008, Bouchy et al. 2008). As measurements accumulate, we expect to be able to confirm (or reject) most of the remaining $\sim 40$ candidates within the next 2-3 years.

\section{Some properties of close-in low-mass planets}

Although these low-mass candidates need to be confirmed, it is tempting to examine their global properties using the preliminary orbital solutions. In particular, comparing the characteristics of the gas giant and low-mass populations, and studying the differences 


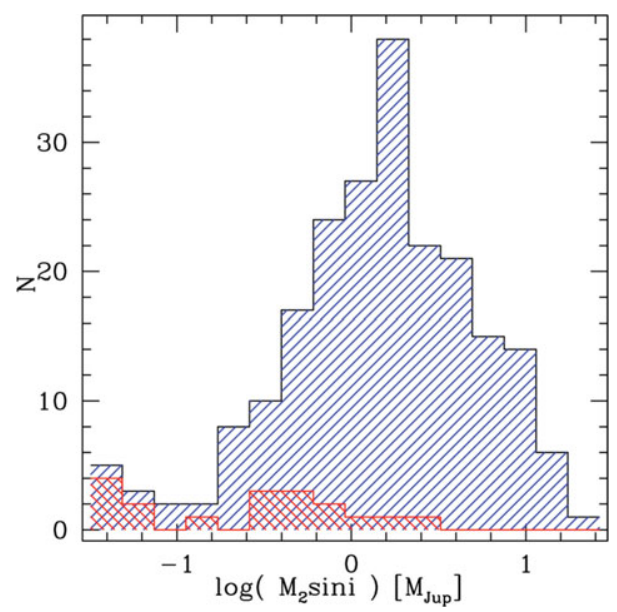

Figure 1. Mass distribution of known extrasolar planets. The recent discoveries of low-mass objects confirm the emergence of a new population below $20-30 \mathrm{M}_{\oplus}$. Published HARPS detections are shown as a cross-hatched histogram.
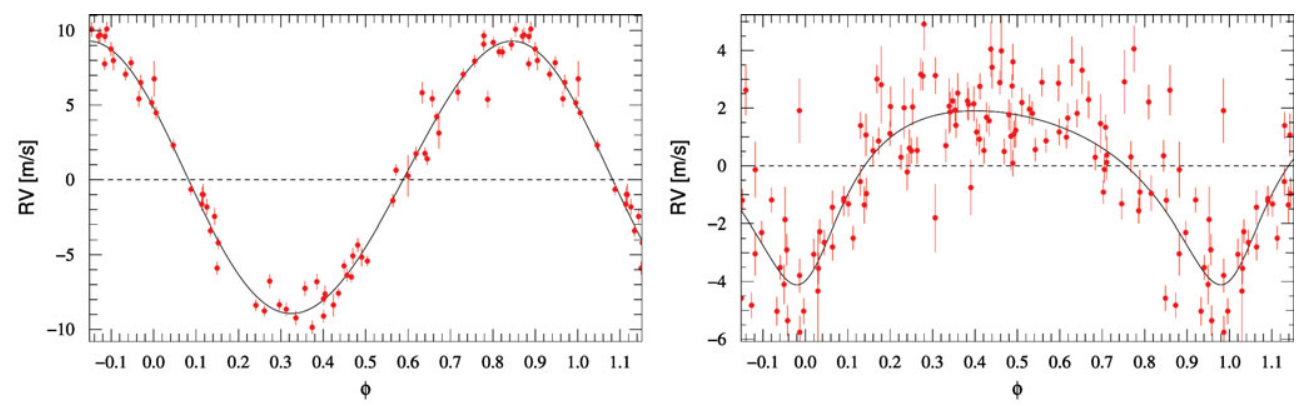

Figure 2. Low-mass planets orbiting HD 47186 and HD 181433 on close-in orbits. HD 47186 b (left) has an orbital period of 4.08 days and a minimum mass of $22.8 \mathrm{M}_{\oplus}$, whereas HD $181433 \mathrm{~b}$ (right) has a period of 9.37 days and a minimum mass of $7.6 \mathrm{M}_{\oplus}$. Both objects are part of multi-planet systems, with at least one giant planet orbiting at larger distances (see Bouchy et al. 2008).

between them, will be highly valuable to constrain planet formation models. The following trends seem to emerge from our sample of low-mass candidates:

- From a uniformly-observed subsample of stars, we estimate that the fraction of stars having planets with minimum masses between $\sim 5-30 \mathrm{M}_{\oplus}$ and orbital periods below 50 days may be as high as $\sim 30 \%$. If confirmed, this number will have a large impact on our perception of planetary systems in general, and Earth-like planets in particular.

- About $80 \%$ of the candidates are found in multi-planet systems.

- After going through a minimum at $\sim 30-40 \mathrm{M}_{\oplus}$, the mass distribution grows towards lower masses with a peak around $10 \mathrm{M}_{\oplus}$, which is most probably due to the detection bias of the technique.

- The period distribution seems to differ from the one of the gas giant population in that the peak is located at larger periods ( $\sim 10$ days) instead of $\sim 3$ days.

- High eccentricities seem common, as for gas giants. 
All these emerging characteristics will help us to better understand several physical processes at work during planet formation, such as the different accretion phases, migration phenomena, dynamical interactions between protoplanets, etc.

\section{The case for a systematic space-based follow-up}

The main question regarding low-mass planets is about their composition. Various combinations of iron, silicates, ices and hydrogen are possible for a given mass, and would translate into very different radii for the planet. Searching for transiting low-mass objects will therefore be one of the most exciting fields of research in the coming years. The HARPS candidates already provide more than 15 top-priority targets for transit search, with transit probability above $\sim 5 \%$ ( $\mathrm{P}<10$ days). Moreover, these planets orbit around nearby bright stars (typically $\mathrm{V}<8$, d $<30 \mathrm{pc}$ ), which makes an in-depth characterization of their composition and atmosphere possible. This is well illustrated by the wealth of information that has been obtained on the two brightest hot Jupiter systems, HD 209458 and HD 189733.

The main difficulty comes from the expected depth of the transit signal: typically $\sim 1.2$ mmag for ice giants and $\sim 0.5$ mmag for super-Earths. Only space-based photometry is able to detect such low amplitudes. A few space-based observatories have the capabilities to do this, including the Spitzer Space Telescope and the MOST satellite. Most importantly, these facilities are also able to continuously monitor a target for more than 10-20 hours, which is necessary given the expected uncertainties on the transit timing based on the HARPS orbital solutions.

As a conclusion, we would like to stress the potentially huge scientific output of a systematic, space-based follow-up of the HARPS transit candidates. Although the individual probability to observe a transit is low, statistics will help and should in the end lead to the detection of the first transiting super-Earth around a nearby bright star.

\section{References}

Bouchy, F., Mayor, M., Lovis, C., Udry, S., Benz, W., Bertaux, J.-L., Delfosse, X., Mordasini, C., Pepe, F., \& Queloz, D. 2008, A\&A, in prep.

Lovis, C., Mayor, M., Pepe, F., Alibert, Y., Benz, W., Bouchy, F., Correia, A. C. M., Laskar, J., Mordasini, C., Queloz, D., Santos, N. C., Udry, S., Bertaux, J.-L., \& Sivan, J.-P. 2006, Nature, 441, 305

Mayor, M., Udry, S., Lovis, C., Pepe, F., Queloz, D., Benz, W., Bertaux, J.-L., Bouchy, F., Mordasini, C., \& Ségransan, D. 2008, A\&SA, submitted (arXiv:0806.4587)

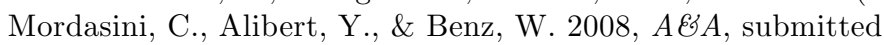

Pepe, F., Correia, A. C. M., Mayor, M., Tamuz, O., Couetdic, J., Benz, W., Bertaux, J.-L., Bouchy, F., Laskar, J., Lovis, C., Naef, D., Queloz, D., Santos, N. C., Sivan, J.-P., Sosnowska, D., \& Udry, S. 2007, A\&A, 462, 769

Santos, N. C., Bouchy, F., Mayor, M., Pepe, F., Queloz, D., Udry, S., Lovis, C., Bazot, M., Benz, W., Bertaux,, J.-L., Lo Curto, G., Delfosse, X., Mordasini, C., Naef, D., Sivan, J.-P., \& Vauclair, S. 2004, A\& A, 426, L19

Udry, S., Mayor, M., Benz, W., Bertaux, J.-L., Bouchy, F., Lovis, C., Mordasini, C., Pepe, F., Queloz, D., \& Sivan, J.-P. 2006, A\& A, 447, 361 